\title{
Spin Depolarization in Quantum Dots
}

\author{
A.S. Sachrajda ${ }^{1}$, C. Gould ${ }^{1,2}$, P.Hawrylak ${ }^{1}$, \\ Y. Feng, ${ }^{1}$ P.Zawadzki, ${ }^{1}$ and Z. Wasilewski, ${ }^{1}$ \\ ${ }^{1}$ Institute for Microstructural Sciences, \\ National Research Council of Canada, Ottawa, Canada K1AOR6 \\ 2 Département de Physique and CRPS, \\ Université de Sherbrooke, Sherbrooke, Canada J1K $2 R 1$
}

Received 27 May, 1999

\begin{abstract}
We have performed single electron spectroscopy experiments on single lateral quantum dots. We demonstrate that the lateral nature of the tunneling as well as the properties of the 2DEG leads can be used to extract new information in the 'spin-flip' regime. Calculations confirm that one needs to go beyond the Hartree-Fock approximation and include correlation effects to explain the experimental observations. The results are found to be consistent with the presence of spin depolarization events at the 'spin-flips'.
\end{abstract}

Quantum dots are often referred to as 'artificial atoms' [1]. This comparison originates from many analogies that exist between the two, e.g. in quantum dots the nucleus is replaced by the confining potential, the ionization energy by the charging energy, etc... Unlike real atoms, however, these parameters are not fixed in dots but may be controllably varied. Performing spectroscopy on these dots is therefore of great fundamental importance and interest. In particular it is important to understand how the properties of quantum dots differ from those of a simple non-interacting puddle of electrons and to understand the influence of the dots geometry and environment. It should also be remembered that what is experimentally observed is dependent not only on the properties of the quantum dot but is also perturbed by the measuring technique. In optical techniques a second carrier species is introduced while in transport techniques the 'leads' play an important role. In this paper we review some experiments and calculations in which transport spectroscopy has been performed on individual few electron lateral quantum dots. We concentrate in particular on the spin-flip regime. The experimental results indicate that one must go beyond even the Hartree-Fock model to explain the results. The results are consistent, however, with exact diagonalization calculations which reveal that as a result of correlations there exist sequences of spin depolarization events at each "spin flip".
The devices used in our experiments are lateral quantum dots defined in a two dimensional electron gas by electrostatic gates. The inset in Fig. 2 shows a micrograph of a device similar to one of the types used. More details on the device design are presented elsewhere [2]. We stress that prior to these devices lateral dots were limited to 40 electrons or more whereas with our design we have achieved lateral dots containing fewer than 10 electrons. Three gates are used to define the quantum dot and the potential barriers whilst a narrow gate placed at the apex of the triangle is used as the third terminal of the Coulomb blockade transistor. McEuen et al.[3] first demonstrated that spectroscopy could be performed on quantum dots by following the third terminal gate voltage position of the Coulomb blockade peak. Tunneling through the quantum dot can only occur when the chemical potential of the dot is matched to that of the reservoirs (i.e.leads). Since the bottom of the conduction band in the dot can be continuously varied by the gate voltage applied to the third terminal, the voltage value of the Coulomb blockade peaks at each magnetic field in the quantum Coulomb blockade regime provides the addition spectrum. Prior to our measurements, however, such techniques have only been applied to vertical quantum dots[4] in the few electron regime. Since vertical devices are capable of studying numerous levels simultaneously including the first one (which provides the crucial calibration infor- 
mation regarding the number of electrons in the dot), the question of what additional information lateral dots can potentially provide needs to be answered. The four most significant advantages of lateral devices are: (1) lateral devices provide direct spatial information, since the electron is tunneling from the edge of the disk-like quantum dot, (2) the electron reservoirs in this case are 2DEG's or more precisely the 2DEG edge - this can be made use ( $c f$. below) to inject only a single spin species, (3) the measurements are also sensitive to the chemical potential of the leads (i.e. the quantum dot can itself be used a local probe of the 2DEG edge) and (4) electronhole asymmetries can be studied in detail in excitation level spectroscopy since electrons can only be added to the edge but can be removed from the center. In this paper we make use of points (1) and (2) to study the spin-flip regime. The consequences and applications of (3) and (4) will be described elsewhere.

The lithographic shape of the dot is triangular. The degeneracies which result in the shell structure of real atoms is related to symmetries which are absent in a hard wall triangular geometry. In the open dot regime i.e. where the dot barriers have a conductance $>2 \mathrm{e}^{2} / \mathrm{h}$ and screening effects are weak we have indeed found evidence that the shell structure is modified. Detailed results in this regime have been submitted elsewhere [5].

Fig. 1 illustrates the 'amplitudes' of several Coulomb peaks (in the 10 to 20 electron regime) from zero magnetic field $(\mathrm{B}=0)$ up to the field at which filling factor 2 is reached in the dot (i.e. the spin unpolarized quantum dot). Remarkably we find more qualitative information in these amplitude plots than from the more usual peak position spectroscopy. It is clear from a comparison with the peak position data that the Coulomb blockade peak amplitude changes reflect ground state level crossings. For the purpose of this paper we note that close to the $\nu=2$ transition the amplitude of the levels with a 'horizontal' tendency (marked ' $\mathrm{H}$ ' and 'L')alternate between neighbouring peaks. These correspond to levels which will condense to the lowest two spin resolved Landau levels. The origin of this remarkable amplitude modulation lies in the 2DEG leads. The dot barriers form edges in the 2DEG. As spin becomes resolved in the 2DEG only the edge state closest to the dot provide electrons for tunneling. Since this is lowest Landau spin down level only spin down electrons are available for tunneling. Thus we observe a 'spin-blockade' effect in which the tunneling amplitude is dramatically reduced for spin-up levels in the dot. We stress that this amplitude modulation is a consequence of the lateral nature of the measurements. The same amplitude modulation occurs in the spin flip regime (i.e. in the transition from a spin unpolarized to a spin polarized quantum dot). At higher magnetic fields in this regime a second amplitude modulation mechanism is also active. Once the magnetic field is raised so that the dot is partially spin polarized the spin up orbitals are near the center of the dot whereas the relevant spin down orbitals are near the edge. Since the tunneling into the dot takes place at the edge this feature also works to reduce the current amplitude for spin up levels. However it is important to note that amplitude modulation is present throughout the spin-flip regime as mentioned above. We can therefore identify the number of electrons in the dot by counting the number of spin flips i.e.by counting the number of amplitude modulation events. We have confirmed that as expected the number of spin flips changes by one for every two levels in our electron number regime. Using this technique we have found that we are able to study dots with fewer than 10 electrons. We also find that based on this electron number allocation, the gate voltage low field spectrum from $\mathrm{B}=0$ to $\nu=2$ is in close agreement with the published vertical circular dot spectrum (nb. if electron-electron interactions and geometry are important then we should not expect exact agreement).

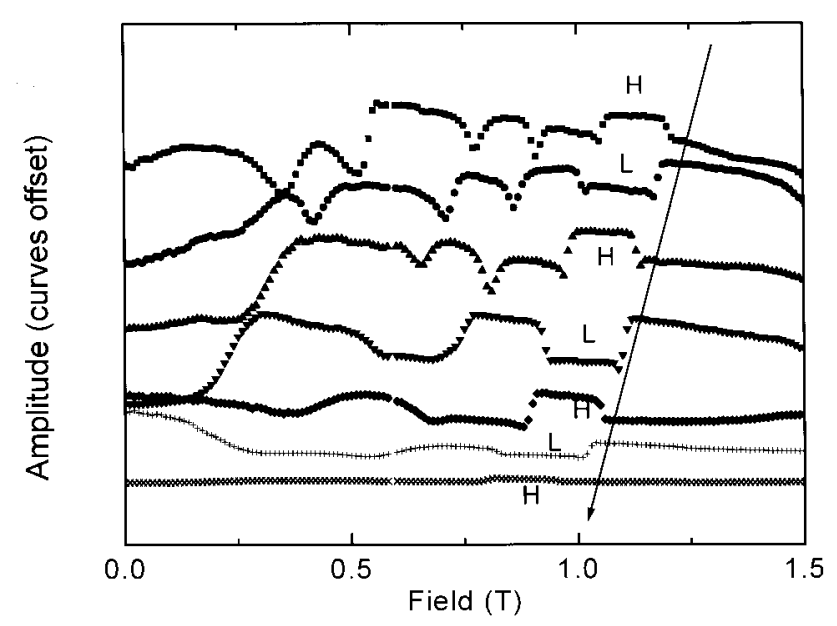

Figure 1. The amplitude of seven peaks versus magnetic field in the low field region. The peaks are in the 10 to 20 electron range. The long arrow marks $\nu=2$ transition to a spin unpolarized dot. An alternating high/low (H/L) amplitude sequence can be seen in the amplitude near this transition (see text for details).

The spin flips in Fig. 2 occur at relatively low mag- 
netic fields. The middle curves illustrate the $\mathrm{CB}$ gate voltage positions (shifted together for clarity) for a dot containing 15-16(lower curve) and 16-17(upper curve) electrons. The amplitude of the peaks is also plotted. It is seen that the amplitude is reduced between the upward and downward cusps. This is the field regime where qualitatively for all models, there exists a difference in the number of spin up electrons between the two relevant ground states associated with that particular Coulomb blockade peak (the CB peaks mark the transition between an $\mathrm{N}$ and $\mathrm{N}+1$ electron dot). It is crucial to note that if only the bare Zeeman energy is assumed to drive the spin flips they would occur at much higher magnetic fields. Klein et al. [6] demonstrated that to account for this as well as the spacing sequence between spin flip events one needs to go to models evoking the Hartree-Fock approximation. The spin flips are in fact driven by electron-electron interaction effects. As the magnetic field is increased the dot effectively shrinks. To counteract the resultant increase in Coulomb energy the dot needs to grow. This is achieved through the spin flip events during which the spin up electrons near the center of the dot move to empty spin down orbitals near the edge of the dot. While the Hartree-Fock model explains the field position of the spin flips a careful examination of Fig. 2 indicates that we need to go beyond this level of approximation. The right insert of the figure presents the temperature dependence of the amplitude of the peaks. It is found experimentally that the amplitude modulation diminishes very rapidly as the temperature is increased and has virtually disappeared by only $200 \mathrm{mK}$, a temperature several times lower than even the bare Zeeman energy for these fields. The lower curve in Fig. 2 plots the ratio of the amplitude of the CB peaks at base temperature and $200 \mathrm{mK}$. It can be seen that the peaks even grow (i.e. the ratio $>1$ ) in the amplitude reduced regions whereas elsewhere they shrink as expected [7]. We note, however, that by invoking exchange the gap, for flipping a spin, is no longer the bare Zeeman gap but is dramatically enhanced. In fact, one would expect the amplitude modulation to persist up to several Kelvin in sharp contrast to the experimental observation. It is interesting to note that in larger dots containing around 50 electrons [3] the experimental observations is similar although a comparison with exact calculations is impossible for such large dots.

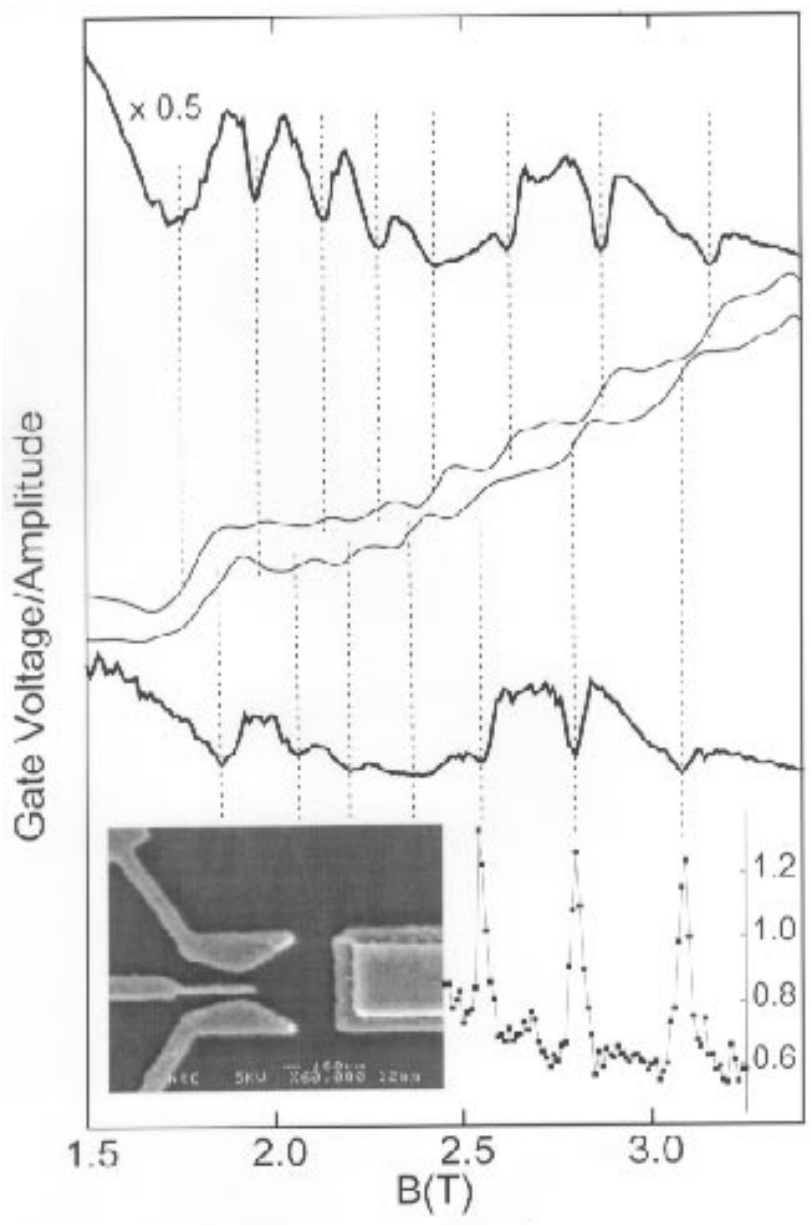

Figure 2. Spin Flips (Experiment): The two curves in the center represent are the gate voltage positions of two Coulomb blockade peaks. They have been shifted together for clarity. They span the 15 to 17 electron quantum dot regime. The bold curves below and above reveal the peak amplitudes. Insets:(1) A SEM of a device similar to one of the two designs used. (2) The ratio of the peak amplitudes taken at $200 \mathrm{mK}$ and $50 \mathrm{mK}$. A value $>1$ indicates that the peak grows as the temperature is raised.

The results of the exact diagonalization calculations are shown in Fig. 3 for the first three spin flips of a dot containing sixteen electrons. To enable exact calculations to be performed on a dot containing sixteen electrons eight electrons near the center of the dot were kept fixed allowing the calculations to be performed for the first four spin flips. Identical theoretical results were obtained for a dot containing eight electrons in which this approximation was removed. Furthermore we have recently observed the same experimental result in a dot containing only eight electrons. More details of the theoretical technique are given elsewhere [2]. A significant consequence of including correlations is observed in the center graph of Fig. 3. This plots the number of spin up electrons versus magnetic field. In the Hartree-Fock and lower level approximations the number of spin up 
electrons monotonically reduces to zero as the field is increased. In the exact scenario, by contrast, whilst the number of spin up electrons does go down after each spin flip event is completed, dramatic spin depolarization events accompany each spin flip. In effect it is seen that a spin flip process is more intricate than the mere removal of a spin up electron near the center of the dot and its replacement as a spin down electron near the

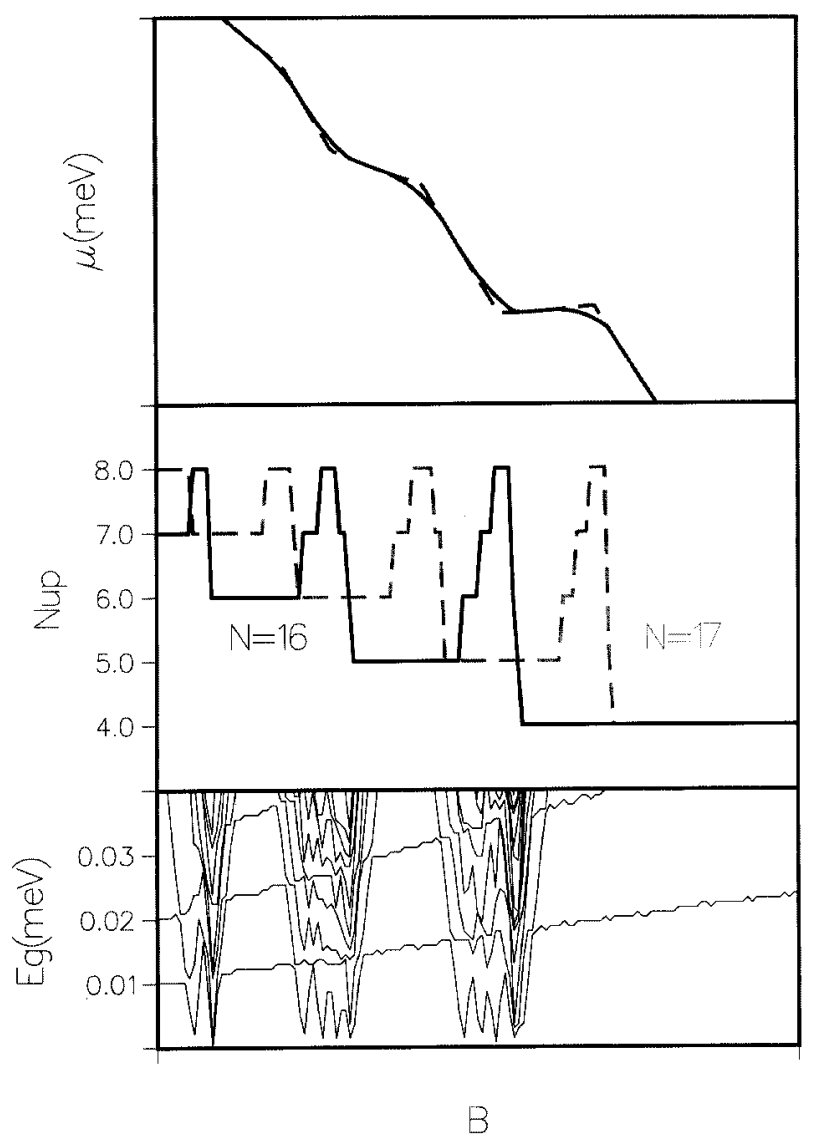

Figure 3. Spin Flips (Theory): Top - Exact(solid) and HF(dashed) chemical potential of the 16 electron dot undergoing spin flips as a function of B. Middle - The number of spin up electrons in the 16 (solid) and 17 (dashed) electron droplet. Bottom - Excited states of the initial droplet.

edge. The interactions of this spin up electron with the background of spin down electrons away from the center and near the edge needs to be considered. How can we experimentally observe these dramatic spin texture effects? In the top graph of Fig. 3 the chemical potential of the dot is plotted against magnetic field. It is found that the only difference between the Hartree-Fock and the exact results which include correlation effects is a slight curving at the cusps. Realistically this confirms that the gate voltage position of the Coulomb blockade peaks cannot be easily used to search for correlation effects. In lateral devices, however, we also have amplitude modulation effects as described above. The lower graph in Fig. 3 shows the excitation spectrum of the dot. By including these correlation effects we have not only recovered the Zeeman gap but have also found excited levels related to the spin textures. These occur at a lower energy than even the bare Zeeman gap. Thus the observation of the quenching of the amplitude modulation in Fig. 2 at very low temperatures is a direct consequence of the presence of the spin textures. Results of excitation level spectroscopy in the same regime will be published elsewhere.

In summary we have studied spin flips in few electron lateral quantum dots. We have compared our experimental results with exact calculations which take correlations into account. In particular it is found that the temperature dependence of the amplitude modulation at spin flip events provides direct evidence of the existence of spin textures in quantum dots.

We wish to acknowledge useful conversations with Allan MacDonald, Guy Austing, J.J.Palacios and Igor Zozoulenko.

\section{References}

[1] P.L. McEuen Science 78, 1729 (1997).

[2] P. Hawrylak, C. Gould, A. Sachrajda, Y. Feng and Z. Wasilewski Phys. Rev. 65, 2801 (1999).

[3] P.L. McEuen, E.B. Foxman, U. Meirav, M.A. Kastner, Y. Meir, N.S. Wingreen and S.J. Wind, Phys. Rev. Lett. 66, 1926 (1991); P.L. McEuen, E.B. Foxman, J.M. Kinaret, U. Meirav, M.A. Kastner, N.S. Wingreen and S.J. Wind, Phys. Rev. B45 11419 (1992).

[4] S. Tarucha, D.G. Austing, T. Honda, R.J.van der Hage, L.P. Kouwenhoven, Phys. Rev. Lett. 77, 3613 (1996).

[5] I.V. Zozoulenko, A.S. Sachrajda, C. Gould, K.-F. Berggren, P. Zawadzki, Y. Feng and Z. Wasilewski, submitted to Phys. Rev. Lett.

[6] O. Klein, C.de C. Chamon, D. Tang, D.M. AbuschMagder, U. Meirav, X.-G. Wen, M.A. Kastner and S.J. Wind, Phys. Rev. Lett. 74, 785 (1995); O. Klein, D. Goldhaber-Gordon, C.de C. Chamon, M.A. Kastner, Phys. Rev. B 53, R4221 (1996).

[7] C.W.J. Beenakker, Phys. Rev. B 44, 1646 (1991). 